\title{
Applications of Cascading Nonlinear Optics to All-Optical Devices
}

\author{
George I. Stegeman, Roland Schiek, Yongsoon Baek and Gijs Krijnen† \\ CREOL, University of Central Florida, P.O.Box 162700 \\ 4000 Central Florida Blvd., Orlando, F1 32816-2700 \\ I. Baumann and W. Sohler \\ Angewandte Physik, Universitat- Gesamthochschule- Paderborn \\ Warburger Strasse 100, D-33098 Paderborn, Germany
}

Current address:

$\ddagger$ Lightwave Devices Group, University of Twente, 7500 AE, Enschede, The Netherlands

Cascading is the process by which second order nonlinearities, when used near phasematching for parametric mixing processes such as second harmonic generation (SHG), can be used to mimic some effects normally associated with third order nonlinearities, more specifically with an intensity-dependent refractive index. The fundamental beam on transmission through a $\chi^{(2)}$-active medium has the form:

$$
E_{0}(\omega)=\frac{1}{2}\left|a_{0}(\omega, z)\right| \sqrt{\frac{2}{c n \epsilon_{0}}} \exp \left[i\left(\omega t-k z-\phi^{N z}(z)\right)\right]+c . c .
$$

away from the phase-matching condition there is a nonlinear phase shift $\phi^{\mathrm{NL}}(\mathrm{z})$ induced whose magnitude and sign depends on the detuning from phase-matching. (There are also interesting effects associated with the amplitudes of the fundamental and second harmonic, including the formation of solitary waves - these will not be discussed here.) It is well-known that nonlinear phase shifts in general are needed for all-optical switching devices and in this paper we will describe the application of such cascaded phase shifts to a fully integrated nonlinear directional coupler and Mach-Zehnder interferometer.[1-4]

The devices utilized Type I phase matching in a $50 \mathrm{~mm}$ long Titanium indiffused symmetrical channel waveguide with propagation along the $\mathrm{X}$-axis on a $\mathrm{Y}$-cut $\mathrm{LiNbO}_{3}$ crystal. Temperature tuning is required at $1320 \mathrm{~nm}$ to implement SHG and the experiments were conducted in an oven around $340^{\circ} \mathrm{C}$. The key to the successful implementation of devices in these waveguides was the temperature distribution in the oven (uniform in the middle and decreasing towards the input and output windows) which allowed large phase shifts $(>1.5 \pi)$ to be obtained with minimal net losses in the fundamental throughput due to SHG $(\sim 10 \%)$. [2]

Both a nonlinear directional coupler (NLDC) and Mach-Zehnder interferometer (NMZI) were made on a $\mathrm{LiNbO}_{3}$ substrate. $[3,4]$ The modelling of the devices was complicated by the presence of four coupled fields and the spatial distribution of the wavevector mismatch was optimized so that SHG is minimized at both outputs simultaneously. The final designs were fabricated and tested with a Nd:YAG laser operating with 90psec pulses. The results are shown in Figs. 1 and 2. For the MZI it is clear that as the input power is increased, the throughput is modulated between $>80 \%$ to less than $20 \%$ of the input. Similarly, for the NLDC, the switching 
from the CROSS to the BAR states with increasing power is clear.

Fig. 1: Measured transmittance of a $\mathrm{LiNbO}_{3}$ integrated Mach Zehnder interferometer as a function of the peak input power with initial phase difference of $\approx \pi$ between the two arms (solid curves, experiment; dotted curves, theory).

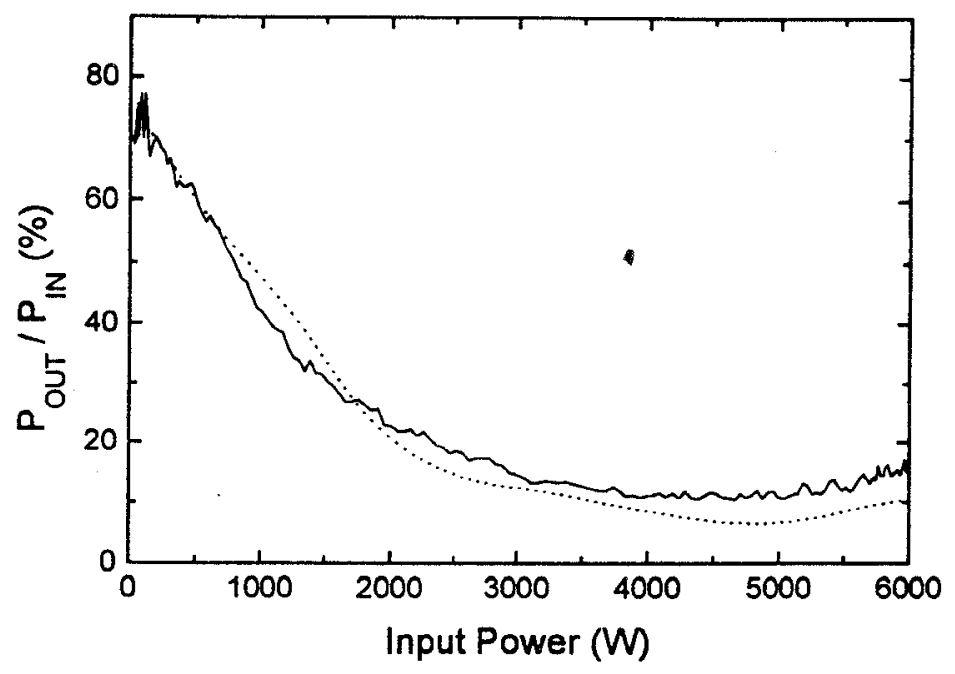

Fig. 2: Power-dependence of a NLDC fundamental output for a crystal temperature of $343^{\circ} \mathrm{C}$. Smooth curves are theory.

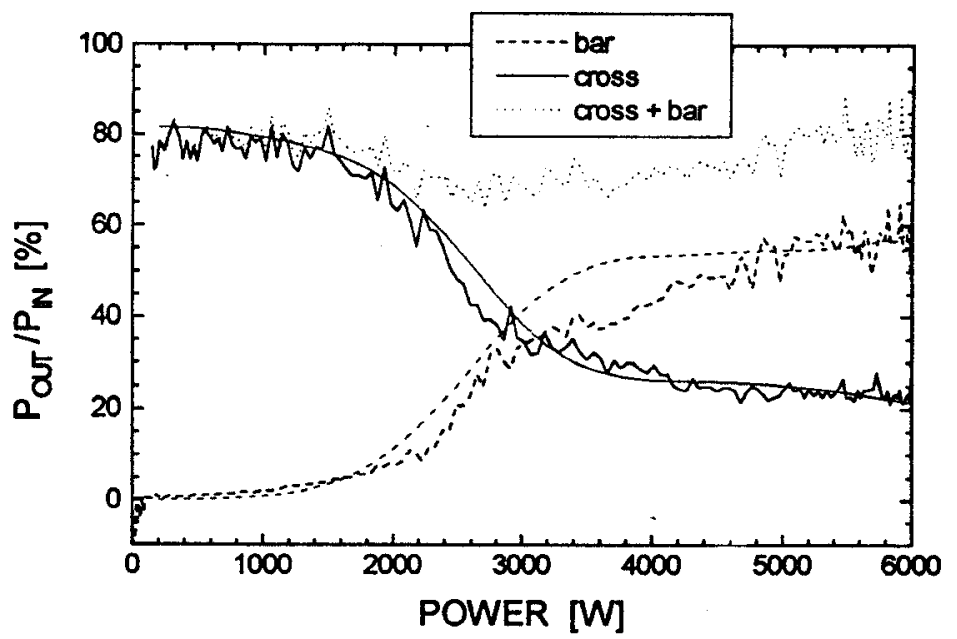

The switching powers in these two applications are in the $\mathrm{KW}$ range. These waveguides have large modal areas $\left(\mathrm{A}_{\text {eff }} \approx 75 \mu \mathrm{m}^{2}\right)$ and utilize small second order nonlinearities $(5 \mathrm{pm} / \mathrm{V})$. There are materials now available with $\mathrm{d}^{(2)} \mathrm{s}$ of $600 \mathrm{pm} / \mathrm{V}$, and in which waveguide containment can be optimized. The switching power varies as $\left|\chi^{(2)}\right|{ }^{2} \mathrm{~A}_{\text {eff }}$ and we expect that milliwatt switching powers will ültimately be possible.

\section{References:}

[1] G.I. Stegeman, SPIE Proceedings on Nonlinear Optical Properties of Advanced Materials, 1852, 7 (1993).

[2] R. Schiek, M.L. Sundheimer, D.Y. Kim, Y. Baek, G.I. Stegeman, H. Suche and W. Sohler, Opt. Lett., 19, 1949 (1994).

[3] R. Schiek, Y. Baek, G. Krijnen, G.I. Stegeman, I. Baumann and W. Sohler, Opt. Lett., in press

[4] Y. Baek, R. Schiek, G. Krijnen, G.I. Stegeman, I. Baumann and W. Sohler, Appl. Phys. Lett., 68, 2055 (1996) 